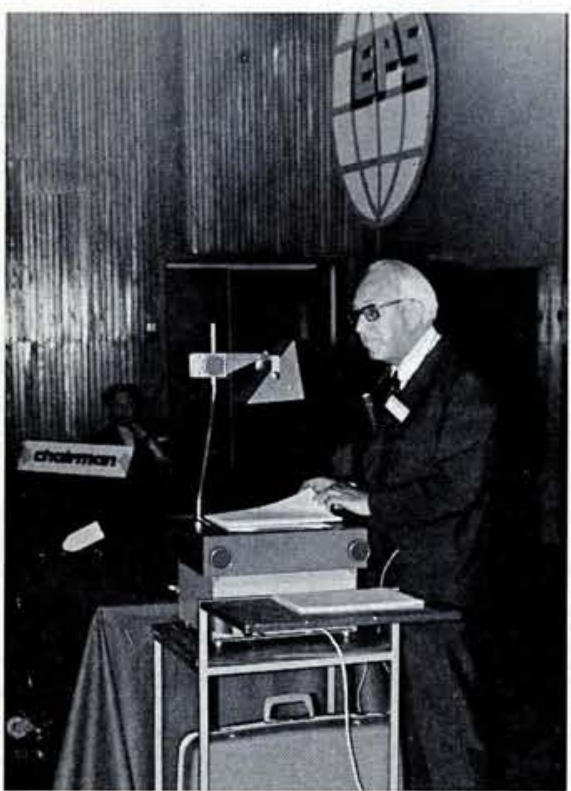

Quantum electronics cont.

multiphoton absorption, coupling constants for near-resonant processes (Raman, etc.) induced birefringence, optical activity and many more. This work at present concentrates on gases being particularly "clean systems" but imporiant experiments have been made also with liquids and solids. On the other hand, nonlinear mixing becomes an increasingly useful tool for the generation of new and tunable frequencies, in particular in the uv and ir.

The availability of pulsed transformlimited tunable dye lasers has allowed considerable progress in the experimental observation of coherent resonant phenomena including multisoliton effects and focusing when the carrier frequency is at exact resonance. Coherent multiphoton processes are now being investigated, as well as fluorescence and resonance scattering which promises to yield considerable information on collisional processes.

The translation of electrotechnical building units to the optical regime has created the subdiscipline of " optical electronics". Reports on ir antennas, distributed feedback lasers, tunnel junctions, heterodyne detection, and integrated optics gave an overview of this field.

Very interesting are the aspects of laser chemistry : detection and control of chemical reactions, for instance, the synthesis of alkalihydrides from vapour ("laser snow"), molecular dissociation (isotope separation, see above) or, very important, the interaction with biological molecules. Important contributions may be expected from this interdisciplinary field in the near future.

D. Pohl

Professor P. Brix giving the first invited lecture at the opening session chaired by the Chairman of the N.P. Division of the EPS Dr. C. Van der Leun.

\title{
Radial Shape of Nuclei
}

Although the 2nd Nuclear Physics Divisional Conference of the EPS held in Cracow, 22-25 June 1976, was not called so officially, it was really a jubilee conference. 65 years have passed since, in 1911, Rutherford with his collaborators discovered the radial dimensions of nuclei, 25 years from 1951 when Lyman, Hanson and Scott pushed forward our knowledge on the radii of charge distribution in nuclei using fast electrons, and 20 years since the famous Hofstadter paper was published in 1956 in Rev. Mod. Phys.

Organized by the Institute of Physics of the Jagellonian University and the Cracow Institute of Nuclear Physics under the chairmanship of Professor A. Budzanowski the Cracow Conference, summarized our understanding of nuclear charge and matter distributions, especially since the International Conference on Electromagnetic Sizes of Nuclei, 1967 in Ottawa, the last conference on this subject. (The First Nuclear Physics Divisional Conference of the EPS was held in Harwell, 24-26 March 1975.)

The first invited talk by P. Brix of Heidelberg illustrated well the progress made since the time when 20 years ago, Hofstadter stated in his paper that "the experiments are just scratching the surface". Now comparison of charge distributions in neighbouring isotones or isotopes gives direct experimental information on the squares of the single-particle radial wave functions of nucleons.

When penetrating deeply into the interior of nuclei we meet a fundamental problem of the validity of quantum electrodynamics at very small distances, say $10 \%$ of the radius of the nucleus, i.e. of the order of $5.10^{-15}$ $\mathrm{cm}$. R. Hofstadter in a special lecture presented the experimental details and results of the most recent Stanford $\mathrm{e}^{+}-\mathrm{e}^{-}$collision experiment proving this validity down to distances of the order of $2.10^{-15} \mathrm{~cm}$. For much deeper insight into the proton structure this limit should still be pushed down to distances of about $8.10^{-16} \mathrm{~cm}$.

Not only the distribution of charge but also of higher electric and magnetic moments of nuclei are now investigated with an increasing accuracy as it was shown in the lecture of R. Eng- fer of Zurich. Another Zurichois, H. K. Walter from SIN in Villigen presented the actual status of our knowledge of the size of nuclei in excited states but pointed out some stagnation in this field of research.

In order to extract information on radial shapes of nuclei we should not only have sufficiently powerful tools at our disposal but also be convinced of the validity of our understanding of interaction. It is also very essential to interpret the data in the proper, umambiguous way. Y.N. Kim of Texas Techn. University has shown how, using some series expansions, the information about charge and matter distribution could be represented in a way independent of some particular model for the distribution function. However, such a reprensentation is unable to solve the problem of the very deep nuclear interior. The predicted bubbles in the form of a large depression of density near the centre of some nuclei, like ${ }^{36} \mathrm{Ar}$, do not seem incompatible with the existing experimental data.

The problems of the theoretical approach to matter distribution in nuclei on the basis of many body theory were covered in the lecture of J.W. Negele of MIT. The speaker pointed out that the present status of theory seems good enough to be used as a quantitative tool in understanding strong interactions in nuclei and not only to extract information on the distribution of nucleons.

The charge distribution in nuclei is still known much better than the matter distribution. D.F. Jackson of Guildford (England) and $\mathrm{H}$. Rebel of Karlsruhe have shown that according to our present knowledge, coming from investigations of hadron interactions with nuclei, any difference between proton and neutron rms radii of nuclei is small, of the order of at most 0,2 $\mathrm{fm}$, which however, is not inconsistent with the possibility of the existence of quite large neutron excess in extreme surface.

Do clusters exist in nuclei ? V. Neudatchin of Moscow presented a theoretician's point of view on this subject, while K. Grotowski of Cracow proposed some possible experimental evidence, especially connected with so called ALAS (anomalous large angle 
scattering) of alpha particles. Grotowski concluded his talk with the remark that one can believe or not in the existence of clusters or nuclear quasimolecules as in other mysterious problems, e.g., the existence of an abominable snowman (Yeti). The speaker belongs evidently to the believers as he illustrated his remark with some beautiful photographs taken in the
Himalayas by the Polish Lothse Expedition with distinct footprints of Yeti in the snow.

The Cracow Conference not only provided a summary of our present state of knowledge of radial shapes of nuclei but also many suggestions for further research in accordance with G. Chr. Lichtenberg's sentence quoted by Brix in his lecture, which can be treated as the golden rule of all experimental sciences, that "one has to make something new to see something new ".

(The Conference abstracts are published as Volume IE in the EPS Conference Abstracts series.)

\section{A. Strzalkowski}

\section{Society News}

\section{Solar Physics Section}

A Solar Physics Section has now been established within the Astronomy and Astrophysics Division of the EPS. The aims of this new Section are :

to promote studies in solar physics and related topics, to facilitate contacts between members, to encourage collaboration in scientific research and exchange of personnel between institutions, to stimulate the interest of young scientists in the field of solar physics, to organize regular meetings, to maintain contacts with related groups within the EPS and to communicate with similar organizations in other parts of the world.

The Solar Physics Section has its roots in two organizations of solar astronomers that have been functioning during the past several years. A number of solar optical physicists associated themselves in 1970 in the Joint Organisation for Solar Observatories (JOSO) which aims at providing for a cooperative solar observatory to be established at a site specially selected for high resolution observations of the solar surface. A number of solar radio astronomers organized themselves in 1971 in the Committee of European Solar Radio Astronomers (CESRA), which has as its purpose to stimulate cooperation, to promote common research programmes and to explore the need and possibility to launch a major common research project.

Both groups held several meetings. JOSO reported its activities in annual reports and CESRA in edited proceedings of its meetings. The two organizations had a combined meeting in Berne, Switzerland, in March 1974 (see $E N$, May 1974) when it was decided to organize a European Solar conference and this meeting was held in Florence, 25-27 February 1975 (its proceedings have been published as Fascicolo 105 of Osservazioni e Memorie dell Osservatorio Astrofisico di Arcetri). On that occasion a new "Organizing Committee of European Solar Meetings" (ESMOC) was elected with C. Jordan (then in Abingdon, now in Oxford) as Chairman and A.D. Fokker (Utrecht) as Secretary. It was agreed that ESMOC should for the present concentrate on the organization of workshops and that it should prepare the formation of a Solar Physics Section of the Astronomy and Astrophysics Division of the EPS.

The reason for creating a Solar Physics Section is the special position solar research takes in between certain physical disciplines and a number of astrophysical fields of research. A Solar Physics Section should bring together physicists and astrophysicists who are interested in problems in which solar physics is involved. Solar Physics is tied with strong links to physical disciplines such as atomic physics, radiative transport, aerodynamics, magnetohydrodynamics and plasma physics. The sun is the nearest cosmical laboratory in which processes relevant to several (astro) physical problems can be studied in detail. Organizing solar physicists within the EPS aims at achieving greater contact across disciplines.

The Secretary of the preliminary board, A.D. Fokker, is now drawing up a list of all those who are interested in joining the section as EPS members or affiliates, and he should be contacted for further details. Once this list has been made, the provisional board (other members are : Bonnet (Verrières-le-Buisson), Chiuderi (Firenze), Gabriel (Culham), Mattig (Freiburg), Mein (Meudon), Meyer (München), Pecker (Paris), Pick (Meudon), Righini (Firenze), Roxburgh
(London) and Zwaan (Utrecht) will stand down and an election for a Section Board will be held.

Regarding future meetings, at present three workshops are being planned. :

- Two-dimensional radiative transfer in small structures in stellar atmospheres, to be held in Naples, organizer B. Caccin, Sept. 14-16 1976.

- Heliography of coronal active regions, to be held in Bonn, organizer E. Fürst, 1977.

- Aspects of atomic physics relevant to solar research, to be held in Switzerland, organizer M.C.E. Huber, 1977.

A general solar meeting will be held in Toulouse in March, 1978.

\section{Present State of Solar Research}

The meeting in Florence brought out the wide scope of the work on solar physics being carried out in Europe. This includes visible region and infra-red observations of the photosphere and low chromosphere, radio frequency observations of the corona and of the bursts associated with active regions and flares, EUV and X-ray observations of the transition region, corona, active regions and flares, made from rockets and satellites, and solar wind studies, mainly from satellites. The theoretical work ranges from the deep interior of the sun over all layers of the atmosphere through the solar wind. Some of the recent work and future plans have been reported in the Proceedings of the meeting in Florence.

We outline below some areas of solar physics which are relevant to other branches of astrophysics.

The fusion reactions which produce the energy in the solar interior are predicted by current models of stellar structure to give rise to a neutrino flux which is almost an order of magnitude larger than the upper limits obtained from sophisticated observation. This 'case of the missing neutrinos' is stimulating renewed interest in the theories of stellar structure and evolution, since changes to these 